Article

\title{
Qualitative Picture of Scaling in the Entropy Formalism
}

\author{
Hans Behringer \\ Faculty of Physics, University of Bielefeld, D-33615 Bielefeld, Germany \\ E-mail: hans.behringer@physik.uni-bielefeld.de
}

Received: 30 June 2008; in revised form: 27 August 2008 / Accepted: 2 September 2008 / Published: 5 September 2008

\begin{abstract}
The properties of an infinite system at a continuous phase transition are characterised by non-trivial critical exponents. These non-trivial exponents are related to scaling relations of the thermodynamic potential. The scaling properties of the singular part of the specific entropy of infinite systems are deduced starting from the well-established scaling relations of the Gibbs free energy. Moreover, it turns out that the corrections to scaling are suppressed in the microcanonical ensemble compared to the corresponding corrections in the canonical ensemble.
\end{abstract}

Keywords: Critical phenomena, microcanonical entropy, scaling relations, Gaussian and spherical model

\section{Introduction}

In a mathematically idealised way continuous phase transitions are described in infinite systems although physical systems in nature or in a computer experiment are finite. The singular behaviour of physical quantities such as the response functions is related to scaling properties of the thermodynamic potential which is used for the formulation [1, 2]. Normally the Gibbs free energy is chosen for the description although other potentials also contain the full information about an equilibrium phase transition. The Hankey-Stanley theorem relates the scaling properties of the Gibbs free energy to the scaling properties of any other energy-like potential that is obtained from the Gibbs free energy by a Legendre transformation [3]. However, the thermodynamic properties can also be deduced from entropy-like potentials, the Massieu-Planck functions, that are related to energy-like potentials by partial inversions [4]. In this paper the scaling relation of the Gibbs free energy is translated to the entropy which is related to the degeneracy of configurations with respect to macroscopic quantities. The considerations are concen- 
trated on systems with algebraically diverging specific heats. Apart from these considerations the paper also intends to give a self-consistent overview of scaling relations in the entropy formalism in the context of continuous phase transitions.

The description of phase transitions in the entropy or microcanonical formalism has gained growing interest in recent years [5-16, 18]. Apart from the study of discontinuous transitions in spin systems some works also investigated continuous phase transitions in the microcanonical approach $[11,14,16$, $17,19,20]$. In particular, scaling relations for the entropy of the infinite system have been formulated and corresponding finite-size scaling relations where discussed [11]. In the present work the scaling relations of the entropy in the infinite volume limit are explicitly deduced starting from the well-established scaling properties of the Gibbs free energy by carefully taking care of the partial inversion that has to be carried out to relate energy-like and entropy-like thermodynamic potentials. Apart from the study of phase transitions the microcanonical ensemble has also attracted recent interest for investigating properties of systems with long range interactions for which the different statistical ensemble are not equivalent any more [21-26]. Other studies in which the microcanonical formalism has been used recently concern biological systems [27-29].

The paper is organised as follows. In section 2 the basics of the description of thermodynamic properties of infinite systems in terms of thermodynamic potentials are summarised. The scaling properties of the Gibbs free energy at a continuous phase transition point are briefly discussed in section 3. A corresponding scaling relation for the entropy is formulated. In section 4 the scaling relation of the entropy is shown to be a direct consequence of the scaling relation of the Gibbs free energy. This extends the Hankey-Stanley theorem to potentials that are related to each other by inversions. Section 5 contains some comments on the microcanonical description of systems with a logarithmically diverging specific heat and systems with a cusp singularity in the specific heat. Section 6 then discusses briefly the microcanonical scaling relations in the exactly solvable Gaussian and spherical models. The correction to scaling terms are heuristically discussed for the entropy formalism in section 7. It turns out that the corrections are qualitatively suppressed in the entropy formalism. A short summary of the findings of this paper is given in section 8 . The paper is entirely written in the language of ferromagnetic systems.

\section{Thermostatics in the entropy formalism}

The basic quantity in the investigation of the statistical properties of a finite magnetic system with $N$ particles is the density of states

$$
\Omega_{N}(E, M)=\int \mathrm{d} \Gamma_{N} \delta(E-\mathcal{H}(\tau)) \delta(M-\mathcal{M}(\tau))
$$

The Hamiltonian $\mathcal{H}$ provides the energy for any configuration $\tau$ from the phase space $\Gamma_{N}$ of all possible microscopic configurations of the $N$-particle system. The magnetisation $M$ of the configuration $\tau$ is measured by the operator $\mathcal{M}$. The microcanonical entropy density of the finite system with $N$ particles is obtained from the density of states by taking the logarithm

$$
s_{N}(e, m)=\frac{1}{N} \ln \Omega_{N}(e N, m N)
$$


where the energy density is defined by $e=E / N$, the magnetisation density is given by $m=M / N$. The thermodynamic properties of the system in the thermodynamic limit are calculated from the entropy

$$
s(e, m)=\lim _{N \rightarrow \infty} s_{N}(e, m)
$$

Note that for discrete spin systems with discrete energy and magnetisation densities for finite systems the thermodynamic limit $N \rightarrow \infty$ has to be carried out in a more sophisticated way so that the resulting entropy function depends on continuous energy and magnetisation densities [30]. The natural variables of the entropy formalism are the energy and the magnetisation, so that the (inverse) temperature and the magnetic field as conjugate variables are determined by the derivatives: $\beta(e, m)=\partial_{e} s(e, m)$ and $h(e, m) \beta(e, m)=-\partial_{m} s(e, m)$. The response functions of the system are related to the curvature properties of the entropy along the energy and magnetisation direction $[11,16]$.

The thermostatics of an infinite system can be investigated either in the energy representation or in the entropy representation [4]. Starting from the entropy representation $s(e, m)$ of a magnetic system in the thermodynamic limit, the energy function $e(s, m)$ is obtained by partially inverting the entropy $s(e, m)$ with respect to the first variable $e$. From the potential $e(s, m)$ one can obtain any other energy-like potential by a Legendre transformation. The Helmholtz free energy is then given by

$$
f(T, m)=\inf _{s}\{e(s, m)-T s\}
$$

and the Gibbs free energy is obtained by

$$
g(T, h)=\inf _{m}\{f(T, m)+h m\} .
$$

As in the thermodynamic limit the thermostatic potentials have well-defined signs of the curvatures along the natural variables one can rewrite the Legendre transformation with the help of derivatives. For the Helmholtz free energy the reformulation results in

$$
f(T, m)=e(\tilde{s}(T, m), m)-T \tilde{s}(T, m)
$$

where $\tilde{s}$ is obtained by inverting the relation $T=\partial_{s} e(s, m)$. Here $\partial_{s}$ denotes the partial derivative with respect to the variable $s$. In a sloppier way, this Legendre transform may be denoted as $f(T, m)=e(s, m)-T s$ with $T=\partial_{s} e$. The connection between the various thermostatic potentials in the thermodynamic limit is schematically depicted as

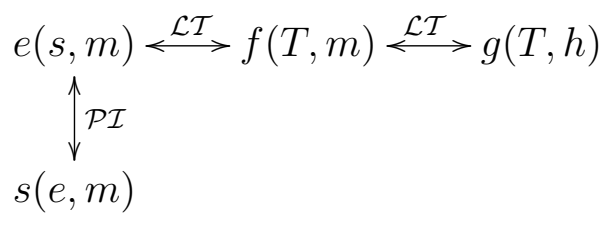

with $\mathcal{L} \mathcal{T}$ denoting a Legendre transformation and $\mathcal{P} \mathcal{I}$ a partial inversion.

The thermal properties of a system can also be investigated in a reduced entropy formalism. Indeed, this formalism has been used extensively in the past to study the behaviour of systems by means of the microcanonical entropy [7-10,12]. The reduced entropy of the infinite system is defined by

$$
s(e)=\lim _{N \rightarrow \infty} \frac{1}{N} \ln \int \mathrm{d} M \Omega_{N}(E, M)
$$


where the energy density $e$ corresponds to the extensive energy $E$ and its conjugate variable (inverse temperature) is $\beta(e)=\partial_{e} s(e)$. The specific heat of the system is determined by

$$
c(e)=-\frac{(\beta(e))^{2}}{\partial_{e} \beta(e)}=-\frac{\left(\partial_{e} s(e)\right)^{2}}{\partial_{e e} s(e)} .
$$

At the critical point $e_{\mathrm{c}}$ the specific heat diverges and is given by the asymptotic law $c(e) \sim|\varepsilon|^{-\alpha_{\varepsilon}}$ where the energy deviation from $e_{\mathrm{c}}$ is denoted by $\varepsilon=e-e_{\mathrm{c}}$ and $\alpha_{\varepsilon}$ is the microcanonical critical exponent of the specific heat. Again, the entropy as a function of the internal energy can be inverted to give the internal energy as a function of the entropy. The internal energy $e(s)$ is again a thermodynamic potential. It is related to the Gibbs free energy by the Legendre transformation

$$
g(T)=\inf _{s}\{e(s)-T s\} .
$$

The relations between the thermodynamic potentials in the reduced formalism are summarised as

$$
s(e) \underset{\mathcal{I}}{\longrightarrow} e(s) \stackrel{\mathcal{L} \mathcal{T}}{\longrightarrow} g(T)
$$

where a Legendre transformation is denoted by $\mathcal{L} \mathcal{T}$ and an inversion by $\mathcal{I}$. The specific heat of the system can also be computed from the Gibbs free energy $g(T)$ and is given by

$$
c(T)=-T \partial_{T T} g(T) .
$$

It has the form $c(T) \sim|t|^{-\alpha}$ near the critical temperature $T_{\mathrm{c}}$. Here the reduced temperature is given by $t=T-T_{\mathrm{c}}$ and $\alpha$ is the critical exponent of the canonical specific heat.

\section{Scaling relations for thermodynamic potentials}

Near the transition point $T_{\mathrm{c}}$ the singular part of the Gibbs free energy satisfies the homogeneity relation

$$
g_{\mathrm{s}}(t, h)=\frac{1}{\lambda} g_{\mathrm{s}}\left(\lambda^{a_{t}} t, \lambda^{a_{h}} h\right)
$$

with a positive re-scaling factor $\lambda[1,2]$. Here the magnetic field is denoted by $h$ and $t$ is again the reduced temperature. The critical exponents are related to the degrees of homogeneity $a_{t}$ and $a_{h}$. The critical exponent $\alpha$ of the canonical specific heat, for example, is given by the expression

$$
\alpha=\frac{2 a_{t}-1}{a_{t}} .
$$

Analogously to the treatment of the Gibbs free energy, the Legendre transforms of the Gibbs free energy - the internal energy $e(s, m)$ and the Helmholtz free energy $f(T, m)$ - can be decomposed into a singular part and a regular background. The singular part is again a generalised homogeneous function [3]. For the internal energy, for example, one has the relation

$$
e_{\mathrm{s}}(\sigma, m)=\frac{1}{\lambda} e_{\mathrm{s}}\left(\lambda^{a_{\sigma}} \sigma, \lambda^{a_{m}} m\right),
$$

with a positive re-scaling factor $\lambda$. The degrees of homogeneity in (15) are given by $a_{\sigma}=1-a_{t}$ and $a_{m}=1-a_{h}$. The reduced entropy $s-s_{\mathrm{c}}$ is denoted by $\sigma$. The homogeneity properties of the MassieuPlanck function entropy $s(e, m)$, which is obtained by partially inverting the internal energy $e(s, m)$, are considered in the next section. 


\section{Entropy formalism and scaling in the infinite system}

Similarly to the scaling relation of the Gibbs free energy the singular part of the entropy satisfies the scaling relation [11]

$$
s_{\mathrm{s}}(\varepsilon, m)=\frac{1}{\lambda} s_{\mathrm{s}}\left(\lambda^{a_{\varepsilon}} \varepsilon, \lambda^{a_{m}} m\right) .
$$

The microcanonical critical exponents can again be expressed in terms of the degree of homogeneity $a_{\varepsilon}$ and $a_{m}$. The exponent $\alpha_{\varepsilon}$, for instance, is given by

$$
\alpha_{\varepsilon}=\frac{1-2 a_{\varepsilon}}{a_{\varepsilon}}
$$

In the following it will be demonstrated that the degree $a_{\varepsilon}$ equals the degree of homogeneity $a_{\sigma}$ introduced in the previous section. The scaling hypothesis (16) for the microcanonical entropy of an infinite system was proposed and discussed in the work of Kastner et al [11].

In the following subsections a heuristic motivation of relation (16) is presented by carefully taking into account the partial inversion that relates energy-like thermodynamic potentials to entropy-like potentials. This partial inversion prevents a direct application of the Hankey-Stanley theorem on Legendre transforms of generalised homogeneous functions. Before the general entropy $s(\varepsilon, m)$ is considered, the reduced entropy formalism is investigated and the results are then utilised to consider the general case. The treatment of the reduced entropy formalism has the additional advantage that it makes the argumentation more transparent.

\subsection{Reduced entropy formalism}

The microcanonical degrees of homogeneity and therefore the critical exponents of the microcanonical singularities are related to their canonical counterparts. This is demonstrated in the following for the entropy $s(e)$ whose singular part fulfils the relation

$$
s_{\mathrm{s}}(\varepsilon)=\frac{1}{\lambda} s_{\mathrm{s}}\left(\lambda^{a_{\varepsilon}} \varepsilon\right)
$$

The starting point of the argumentation is the scaling relation of the Gibbs free energy which is then translated to the scaling properties of the internal energy as a function of its natural variable entropy. To this end, the Legendre transformation between the Gibbs free energy and the internal energy is explicitly worked out. Then the internal energy is solved for the entropy leading to the scaling relation for the entropy as a function of the internal energy.

Consider the canonical specific heat $c^{(\mathrm{c})}$ in the vicinity of the critical point $t=0$. The specific heat with an algebraic divergence at the critical point is given by

$$
c^{(\mathrm{c})}(t)=(1-\alpha) T_{\mathrm{c}} P_{ \pm}|t|^{-\alpha}+c_{\mathrm{b}}(t)
$$

with positive coefficients $P_{ \pm}$, where the subscript plus generally refers to coefficients for positive reduced temperatures (and energies, respectively) and the subscript minus to coefficients for negative reduced temperatures. The exponent $\alpha$ is assumed to be positive and smaller than one. The contribution $c_{\mathrm{b}}$ denotes the background to the singular specific heat. One contribution to $c_{\mathrm{b}}$ arises from the analytic 
part of the Gibbs free energy, but it may also contain singular terms that are characterised by exponents smaller than $\alpha$ so that these additional singular terms become unimportant in the limit $t \rightarrow 0$. These subdominant singular terms are due to the contribution of irrelevant scaling fields to the singular part of the Gibbs free energy. Integrating the relation

$$
c^{(c)}(t)=-T \partial_{t t} g(t)
$$

gives rise to the expression

$$
g(t)=-\frac{P_{ \pm}}{2-\alpha}|t|^{2-\alpha}+g_{\mathrm{b}}(t)
$$

where $g_{\mathrm{b}}$ is again the background to the dominating singular contribution. The Gibbs free energy contains a singular part that satisfies the homogeneity relation

$$
g_{\mathrm{s}}(t)=\frac{1}{\lambda} g_{\mathrm{s}}\left(\lambda^{a_{t}} t\right)
$$

with a positive re-scaling factor $\lambda$. The degree $a_{t}$ is related to the exponent $\alpha$ by relation (14). The background free energy $g_{\mathrm{b}}(t)$ can be expanded into an asymptotic series giving

$$
g_{\mathrm{b}}(t)=s_{\mathrm{c}} t-\frac{Q}{2-\alpha+\theta}|t|^{2-\alpha+\theta}+\ldots
$$

with some coefficients $s_{\mathrm{c}}$ and $Q$. A constant contribution that is allowed in general is chosen to be zero and the correction to scaling exponent $\theta$ is positive and characterises the next leading term in the expansion of $g_{\mathrm{b}}$. This next leading term might be a quadratic term arising from the regular part of the Gibbs free energy. In this case the exponent $\theta$ is just $\alpha$. However additional non-analytic correction to scaling terms also arise from the singular part of the Gibbs free energy. For the universality class of the three-dimensional Ising model the correction to scaling term is described by a non-integer exponent which is approximately given by $\theta \approx 0.5$ [31-33]. In the subsequent considerations it will be argued that the correction term characterised by the exponent $\theta$ is unimportant for the dominant behaviour of the Legendre transform of the Gibbs potential in the vicinity of the critical point.

The positivity of $P_{ \pm}$ensures that the free energy $g$ is concave in the vicinity of the critical point $t=0$. The internal energy $e$ as a thermostatic potential depends on the entropy $s$ and is related to $g$ by the Legendre transformation

$$
e(s)=g(t(s))+T(s) s
$$

where the temperature $T=t+T_{\mathrm{c}}$ as a function of the entropy $s$ is obtained by inverting the relation $s(t)=-\partial_{t} g(t)$. From relations (21) and (23) one obtains the expression

$$
\sigma(t)=P_{ \pm} \operatorname{sgn}(t)|t|^{1-\alpha}+Q \operatorname{sgn}(t)|t|^{1-\alpha+\theta}+\ldots
$$

for the reduced entropy $\sigma(t)=s(t)-s_{\mathrm{c}}$. In the asymptotic regime $t \rightarrow 0$ and therefore $\sigma \rightarrow 0$ expression (25) can be inverted giving the first leading term

$$
t(\sigma)=P_{ \pm}^{\frac{1}{\alpha-1}} \operatorname{sgn}(\sigma)|\sigma|^{\frac{1}{1-\alpha}}+\ldots
$$

Inserting this expression in the functional equation

$$
\sigma\left(t\left(\sigma^{\prime}\right)\right)=\sigma^{\prime}
$$


for the inverse function $t$ of the reduced entropy $\sigma$ one gets

$$
\sigma\left(t\left(\sigma^{\prime}\right)\right)=\sigma^{\prime}+Q\left(\frac{1}{P_{ \pm}}\right)^{\frac{1-\alpha+\theta}{1-\alpha}}\left|\sigma^{\prime}\right|^{\frac{1-\alpha+\theta}{1-\alpha}}
$$

The functional equation (27) for the inverse $t(\sigma)$ is therefore satisfied up to a term of order

$$
\frac{1-\alpha+\theta}{1-\alpha}>1
$$

This shows that the leading correction term characterised by the exponent $\theta$ can be neglected in the vicinity of the critical point no matter which part of the Gibbs free energy it arises from. The Legendre transformation (24) therefore yields

$$
e(\sigma)=T_{\mathrm{c}} s_{\mathrm{c}}+T_{\mathrm{c}} \sigma+R_{ \pm}|\sigma|^{\frac{2-\alpha}{1-\alpha}}+\ldots
$$

in the asymptotic limit $\sigma \rightarrow 0$. The coefficients $R_{ \pm}$are given by

$$
R_{ \pm}=\left(1-\frac{1}{2-\alpha}\right) P_{ \pm}^{\frac{1}{\alpha-1}}=\frac{1-\alpha}{2-\alpha} P_{ \pm}^{\frac{1}{\alpha-1}}
$$

and are thus positive for $\alpha \in] 0,1[$. The entropy is now obtained by inverting the reduced energy $\varepsilon(\sigma)=$ $e(\sigma)-T_{\mathrm{c}} s_{\mathrm{c}}$. The inversion can be done iteratively. First, the leading (lowest order) term is considered separately and the associated inverse function is worked out. Then, the next order term is included in such a way that the functional equation (27) is satisfied up to the next order. This procedure is then iteratively repeated by successively including further higher order terms. In the limit of small reduced entropies $\sigma$ and thus small reduced energies $\varepsilon$ one gets

$$
s(\varepsilon)=s_{\mathrm{c}}+\frac{1}{T_{\mathrm{c}}} \varepsilon-\frac{R_{ \pm}}{T_{\mathrm{c}}}\left(\frac{1}{T_{\mathrm{c}}}\right)^{\frac{2-\alpha}{1-\alpha}}|\varepsilon|^{\frac{2-\alpha}{1-\alpha}}+\ldots
$$

An asymptotic expression of the form (32) was already discussed in the literature without establishing the connection to the Gibbs free energy (21), however [7, 9]. The entropy $\sigma(\varepsilon)=s(\varepsilon)-s_{\mathrm{c}}$ is negatively curved near the critical energy $e_{\mathrm{c}}=T_{\mathrm{c}} s_{\mathrm{c}}$ due to the positivity of the coefficients $R_{ \pm}$.

The asymptotic series (32) shows that the microcanonical entropy in the thermodynamic limit contains a singular part $s_{\mathrm{s}}(\varepsilon)$ as a function of the reduced energy $\varepsilon$ which satisfies relation (18). The degree of homogeneity $a_{\varepsilon}$ is connected to the canonical degree $a_{t}$ by $a_{\varepsilon}=1-a_{t}$. The above considerations show that the scaling law (18) follows from the corresponding relation (22) of the Gibbs free energy.

The microcanonical specific heat that is obtained from relation (32) has the asymptotic form

$$
c^{(\mathrm{m})}(\varepsilon) \sim \frac{1}{\partial_{\varepsilon \varepsilon} s(\varepsilon)} \sim|\varepsilon|^{-\alpha_{\varepsilon}}
$$

in the limit of small reduced energies. Note that the appearance of a quadratic term in the expansion (32) would lead to a cusp singularity in the specific heat. Therefore, a quadratic term must be absent in the asymptotic expansion of $s(\varepsilon)$ for a system with an algebraically diverging specific heat. The exponent $\alpha_{\varepsilon}$ which specifies the divergence of the microcanonical specific heat near the critical energy $e_{\mathrm{c}}$ is related to the canonical exponent by

$$
\alpha_{\varepsilon}=\frac{1-2 a_{\varepsilon}}{a_{\varepsilon}}=\frac{\alpha}{1-\alpha} .
$$

Note that the microcanonical exponent $\alpha_{\varepsilon}$ has not to be confused with a Fisher-renormalised exponent [34]. The Fisher-renormalised exponent of $\alpha$ acquires an additional minus sign compared to (34). 


\subsection{General entropy}

The argumentation for the reduced entropy in the thermodynamic limit is readily extended to a more general entropy that, in addition to the energy, also depends on the magnetisation. The entropy $s(e, m)$ is obtained from the internal energy $e(s, m)$ by carrying out a partial inversion with respect to the entropy variable (see relation 7). An asymptotic expansion of the internal energy that is consistent with the scaling relation (15) is given by

$$
e(\sigma, m)=e_{\mathrm{c}}+T_{\mathrm{c}} \sigma+A_{ \pm}|\sigma|^{\frac{1}{a_{\sigma}}}+B_{ \pm}|m|^{\frac{1}{a_{m}}}+C_{ \pm}|m|^{\frac{1-a_{\sigma}}{a_{m}}} \sigma+\ldots
$$

The asymptotic expression (35) is chosen to reproduce the singular asymptotic behaviour of physical quantities which is deduced from the scaling relation (15). Note, however, that certain properties of the internal energy - for an entropy below the critical point, for instance, it has to be constant for magnetisations in the interval $\left[-m_{\mathrm{sp}}, m_{\mathrm{sp}}\right]$ - are not yet incorporated in (35), but the argumentation to follow still applies to the concave envelop function of (35) with respect to the magnetisation direction. Defining a new (shifted) energy variable

$$
\tilde{\epsilon}(\sigma, m)=\varepsilon(\sigma, m)-B_{ \pm}|m|^{\frac{1}{a_{m}}}
$$

and the new auxiliary variable

$$
\theta_{\mathrm{c}}=T_{\mathrm{c}}+C_{ \pm}|m|^{\frac{1-a_{\sigma}}{a_{m}}}
$$

for fixed magnetisations $m$, the asymptotic series (35) can be re-cast into the expression

$$
\tilde{\epsilon}=\theta_{\mathrm{c}} \sigma+A_{ \pm}|\sigma|^{\frac{1}{a_{\sigma}}}+\ldots
$$

which is of the form of the series (30) and has therefore the partial inverse

$$
\sigma(\tilde{\epsilon})=\frac{1}{\theta_{\mathrm{c}}} \tilde{\epsilon}-\frac{A_{ \pm}}{\theta_{\mathrm{c}}}\left(\frac{1}{\theta_{\mathrm{c}}}\right)^{\frac{1}{a_{\sigma}}}|\tilde{\epsilon}|^{\frac{1}{a_{\sigma}}}+\ldots
$$

for small energies $\tilde{\epsilon}$ and entropies $\sigma$. Plugging in the expressions for $\tilde{\epsilon}$ and $\theta_{\mathrm{c}}$ and considering the limit of small energies $\varepsilon$ and magnetisations $m$, one finally ends up with the asymptotic expression

$$
s(\varepsilon, m)=s_{\mathrm{c}}+\frac{1}{T_{\mathrm{c}}} \varepsilon-\frac{A_{ \pm}}{T_{\mathrm{c}}}\left(\frac{1}{T_{\mathrm{c}}}\right)^{\frac{1}{a_{\sigma}}}|\varepsilon|^{\frac{1}{a_{\sigma}}}-\frac{B_{ \pm}}{T_{\mathrm{c}}}|m|^{\frac{1}{a_{m}}}-\frac{C_{ \pm}}{T_{\mathrm{c}}}|m|^{\frac{1-a_{\sigma}}{a_{m}}} \varepsilon+\ldots
$$

These heuristic considerations show that the entropy surface $s(\varepsilon, m)$ contains a singular part that satisfies the scaling relation (16) with the degree of homogeneity $a_{\varepsilon}=a_{\sigma}=1-a_{t}$. This is again a consequence of the canonical scaling laws for the singular part of the Gibbs free energy.

\section{Transformation from the temperature to the energy variable}

In this section the relation between the microcanonical critical exponents and the corresponding canonical exponents is considered for systems with a specific heat that does not diverge algebraically. A similar treatment can be carried out within a cluster molecular field approximation [35]. Consider first a system whose (canonical) specific heat $c^{(\mathrm{c})}$ near the transition point has the asymptotic form

$$
c^{(\mathrm{c})}(t) \sim c_{0}-P_{ \pm}|t|^{-\alpha}
$$


for small reduced temperatures $t$. If the exponent $\alpha$ is positive the constant contribution $c_{0}$ can be neglected and one has again the case of an algebraically diverging specific heat. For a negative exponent $\alpha$, as it can be found, for example, in the XY-model (for a microcanonical investigation, see [36]) the specific heat has a cusp singularity. In the thermodynamic limit all physical quantities can be expressed as functions of the internal energy. Integrating expression (41) gives

$$
\varepsilon \sim c_{0} t-\frac{P_{ \pm}}{1-\alpha} \operatorname{sgn}(t)|t|^{1-\alpha} .
$$

Hence, the dominating term in the limit $t \rightarrow 0$ is the linear one if $\alpha$ is negative as $1-\alpha=1+|\alpha|>1$. Therefore, one has $\varepsilon \sim t$ near the transition point in case of a negative $\alpha$. Consider now a physical quantity $a$ which behaves as $a \sim|t|^{-\kappa}$ in the canonical description. The microcanonical exponent $\kappa_{\varepsilon}$ of the physical quantity $a$ is identical to the exponent $\kappa$ of the canonical description as $\varepsilon \sim t$ near the transition point. For a positive $\alpha$ on the other hand the dominant term in the expression for the reduced energy is $|t|^{1-\alpha}$ and therefore one has $|t| \sim|\varepsilon|^{\frac{1}{1-\alpha}}$ leading to the renormalised microcanonical exponent $\kappa_{\varepsilon}=\kappa /(1-\alpha)$.

The consideration above only applies to exponents that characterise the temperature and the energy behaviour of thermostatic quantities of a system with a specific heat which has an algebraic divergence or a cusp singularity. For a logarithmically diverging specific heat

$$
c^{(\mathrm{c})}(t) \sim-\ln |t|
$$

the deviation of the internal energy from the transition energy varies as $\varepsilon \sim|t|-t \ln |t|$ in the limit of vanishing $t$. This leads to the expression $t \sim-\varepsilon / \ln |\varepsilon|$ for the reduced temperature in the asymptotic regime $t \rightarrow 0$. The algebraically diverging function $a(t) \sim|t|^{-\kappa}$ hence behaves as

$$
a \sim\left(-\frac{|\varepsilon|}{\ln |\varepsilon|}\right)^{-\kappa}
$$

when expressed as a function of the internal energy. Although the singularity shows a more complex structure in the energy picture its critical exponent $\kappa_{\varepsilon}$ is identical to the exponent $\kappa$ specifying the singular behaviour in the canonical ensemble.

\section{Gaussian and spherical model}

The Gaussian and in particular the spherical model are continuous spin models that can be solved exactly [37-39]. These models are therefore often used to discuss properties of phase transitions analytically. In this section the scaling properties of the microcanonical physical quantities and the relation of the corresponding critical exponents to the canonical critical indices are discussed for these models.

The spin variables $\tau_{i}$ are defined on the $N$ lattice points on a $d$-dimensional hypercube and can take on any real value, i. e. $\tau_{i} \in \mathbb{R}$ for $i=1, \ldots, N$. For both models the Hamiltonian is given by

$$
\mathcal{H}(\tau)=-J \sum_{\langle i, j\rangle} \tau_{i} \tau_{j},
$$

where the sum extends over all nearest neighbour lattice sites and $J$ is the exchange constant. The magnetisation is given by

$$
\mathcal{M}(\tau)=\sum_{i} \tau_{i}
$$


The two models differ by the integral measure which is used to calculated the density of states (1): for the Gaussian model, one has

$$
\mathrm{d} \Gamma_{N}=\prod_{i} \mathrm{~d} \tau_{i} \frac{1}{\sqrt{\pi}} \exp \left(-\tau_{i}^{2}\right)
$$

and for the spherical Model one has

$$
\mathrm{d} \Gamma_{N}=\left(\prod_{i} \mathrm{~d} \tau_{i}\right) \delta\left(N-\sum_{i} \tau_{i}^{2}\right) .
$$

In case of the spherical model the phase space is thus the sphere of radius $\sqrt{N}$ in $\mathbb{R}^{N}$.

In the following subsection the analysis of the critical properties of the specific heat is briefly sketched exemplarily for the Gaussian model. The analysis of the critical properties is carried out along the same lines of reasoning as in the case of the spherical model [43].

\subsection{Gaussian model}

The density of states (1) of the Gaussian model (47) and the corresponding entropy density (3) in the thermodynamic limit $N \rightarrow \infty$ can be calculated analytically by applying a saddle point integration (as in case of the spherical model, for details see $[43,44])$. The entropy density is then obtained to be

$$
s(e, m)=z_{0} e+w_{0} m+-\frac{1}{2} \int \frac{\mathrm{d}^{d} \varphi}{(2 \pi)^{d}} \ln \left(1-z_{0} J \sum_{j} \cos \varphi_{j}\right)+\frac{w_{0}^{2}}{4\left(1-z_{0} d J\right)}
$$

where $z_{0}$ and $w_{0}$ as functions of the energy $e$ and the magnetisation $m$ solve the saddle point equations

$$
\frac{\partial \phi}{\partial z}=e+\frac{1}{2} \int \frac{\mathrm{d}^{d} \varphi}{(2 \pi)^{d}} \frac{J \sum_{j} \cos \varphi_{j}}{1-z J \sum_{j} \cos \varphi_{j}}+\frac{d J}{4(1-z d J)^{2}} w^{2}=0
$$

and

$$
\frac{\partial \phi}{\partial w}=m+\frac{1}{2(1-z d J)} w=0
$$

From applying the saddle point evaluation one also gets the restriction $\left.z_{0} \in\right]-1 /(d J), 1 /(d J)[$. Notice also that the solution of the saddle point equations determine the microcanonical (inverse) temperature $\beta(e, m)=\partial_{e} s(e, m)=z_{0}(e, m)$ and the microcanonical magnetic field $h(e, m) \beta(e, m)=$ $-\partial_{m} s(e, m)=-w_{0}(e, m)$.

In the following the system is considered for zero magnetic field $h(e, m)=0$. This equation $h(e, m)=0$ determines the equilibrium macrostate $\left(e, m_{\mathrm{sp}}(e)\right)$ for a given energy $e$. The corresponding equilibrium temperature is defined to be $\beta_{0}(e)=\beta\left(e, m_{\mathrm{sp}}(e)\right)$. From relation (51) one gets in zero field the equation $2 m_{\mathrm{sp}}(e)\left(1-\beta_{0}(e) d J\right)=0$, which has to be obeyed by the spontaneous magnetisation $m_{\mathrm{sp}}(e)$. This equation has the trivial solution $m_{\mathrm{sp}}(e)=0$. A non-zero spontaneous magnetisation is only possible if the bracket vanishes, i. e. if $\beta_{0}=1 /(d J)$. This, however, is not possible as $\beta_{0}(e)$ has to be in ] $-1 /(d J), 1 /(d J)[$. Thus, the Gaussian model does not exhibit a non-zero spontaneous magnetisation. The temperature $\beta_{0}$ cannot exceed $\beta_{\mathrm{c}}:=1 /(d J)$ which reflects the breakdown of the Gaussian model for low temperatures $[40,42]$. If the Gaussian model possesses a phase transition in zero magnetic field in any dimension $d$ it has to occur at the temperature $\beta_{\mathrm{c}}$. 
With $w_{0}=0$ corresponding to zero magnetic field, one gets from the saddle point equation (50) an expression for the energy $e$ that corresponds to the temperature $\beta_{0}$ :

$$
e=-\frac{1}{2} \int \frac{\mathrm{d}^{d} \varphi}{(2 \pi)^{d}} \frac{J \sum_{j} \cos \varphi_{j}}{1-\beta_{0}(e) J \sum_{j} \cos \varphi_{j}} .
$$

As the specific heat (9) in the thermodynamic limit is positive there exists a one-to-one correspondence between the energy $e$ and the corresponding temperature $\beta_{0}(e)$. This allows the calculation of the energy which corresponds to a given temperature instead of solving the integral equation (52) for $\beta_{0}(e)$ if the energy $e$ is given. In the following only physical temperatures are considered, i. e. $\beta_{0} \in[0,1 /(d J)[$. For the temperature $\beta_{\mathrm{c}}$ one has the energy

$$
e_{\mathrm{c}}=-\frac{1}{2} \int \frac{\mathrm{d}^{d} \varphi}{(2 \pi)^{d}} \frac{d J \sum_{j} \cos \varphi_{j}}{d-\sum_{j} \cos \varphi_{j}}
$$

In the limit $\varphi_{j} \rightarrow 0, j=1, \ldots, d$, the $\operatorname{sum} \sum_{j} \cos \varphi_{j}$ tends to $d$ and thus the vanishing denominator in (53) might cause a diverging integral. For small $\varphi_{j}$ the denominator can be approximated by $\frac{1}{2}\left(\varphi_{1}^{2}+\right.$ $\left.\ldots+\varphi_{d}^{2}\right)$. Introducing polar coordinates for the $d$-dimensional $\varphi$-space and excluding a small sphere of radius $\delta$ from the integration over $\varphi_{j}, j=1, \ldots, d$ one gets the factor $-\lim _{\delta \rightarrow 0} \int_{\delta} \mathrm{d} \varphi \varphi^{d-3}$ to whom the dominant small $\varphi_{j}$ contributions to the integral (53) are proportional. The modulus of the vector $\left(\varphi_{1}, \ldots, \varphi_{d}\right)$ is here denoted by $\varphi$. For one and two dimensions this factor diverges in the limit $\delta \rightarrow 0$ and one has $e_{\mathrm{c}}=-\infty$ which means that the Gaussian model does not undergo a phase transition in these dimensions. For dimensions greater than two the limit $\delta \rightarrow 0$ exists and hence the (critical) energy $e_{\mathrm{c}}$ is finite and the model will have a phase transition. For energies $e$ below this critical energy $e_{\mathrm{c}}$, however, the Gaussian model is not defined for $d>2$. For $d \leq 2$ dimensions the Gaussian model is well-defined in the microcanonical formalism with the energy $e$ as its natural variable.

The specific heat in zero magnetic field can be computed by differentiating equation (50):

$$
c_{0}(e)=-\frac{\left(\beta_{0}(e)\right)^{2}}{\frac{\partial}{\partial e} \beta_{0}(e)}=\frac{\left(\beta_{0}(e)\right)^{2}}{2} \int \frac{\mathrm{d}^{d} \varphi}{(2 \pi)^{d}} \frac{\left(J \sum_{j} \cos \varphi_{j}\right)^{2}}{\left(1-\beta_{0}(e) J \sum_{j} \cos \varphi_{j}\right)^{2}} .
$$

A divergence of the specific heat might again be caused by the contributions to this integral coming from small $\varphi_{j}$. Performing a similar analysis as above one finds that the specific heat diverges at $e_{\mathrm{c}}$ for dimensions $2<d \leq 4$. In dimensions $d>4$ the specific heat of the Gaussian model stays finite.

In the remaining paragraphs of this subsection the character of the divergence of the specific heat in $2<d \leq 4$ dimension is investigated. To this end the variable $\vartheta>0$ is defined by $\beta_{0}(e)=\beta_{\mathrm{c}}-\vartheta(e)$, so that $\vartheta$ measures the deviation of the temperature form the critical temperature $\beta_{\mathrm{c}}$. Defining the integral

$$
R(\vartheta):=-\frac{1}{2} \int \frac{\mathrm{d}^{d} \varphi}{(2 \pi)^{d}} \frac{J \sum_{j} \cos \varphi_{j}}{1-\left(\beta_{\mathrm{c}}-\vartheta\right) J \sum_{j} \cos \varphi_{j}}
$$

one has $\varepsilon=e-e_{\mathrm{c}}=R(\vartheta(e))-R(0)$ for the deviation of the energy $e$ from the critical value $e_{\mathrm{c}}$. For small positive $\vartheta$ one has the asymptotic relations [45]

$$
\varepsilon=R(\vartheta)-R(0) \sim\left\{\begin{array}{cl}
\vartheta^{(d-2) / 2} & \text { if } 2<d<4, \\
-\vartheta \ln \vartheta & \text { if } d=4, \\
\vartheta & \text { if } d>4 .
\end{array}\right.
$$


In the vicinity of the critical energy $e_{\mathrm{c}}$, i. e. for small $\vartheta$, the specific heat is now given by $c_{0}(e) \sim \beta_{\mathrm{c}}^{2} R^{\prime}(\vartheta)$ from which the behaviour for small positive values of $\vartheta$ can be obtained in view of (56). Using relation (56) to express $\vartheta$ as a function of $\varepsilon$ one has therefore the asymptotic relation $c_{0}(\varepsilon) \sim \vartheta^{\frac{d-4}{2}} \sim \varepsilon^{-\frac{4-d}{d-2}}$ for small $\varepsilon$ in dimensions $2<d<4$. The algebraically diverging specific heat is hence characterised by the critical exponent $\alpha_{\varepsilon}=(4-d) /(d-2)$ which is related to the canonical critical exponent $\alpha=(4-d) / 2$ (see, e.g., [40-42]) by the relation $\alpha_{\varepsilon}=\alpha /(1-\alpha)$. For the case $d=4$ one has $c_{0}(e) \sim-\ln \vartheta$ and thus the dominant term in the limit $\varepsilon \rightarrow 0$ is given by $c_{0}(\varepsilon) \sim-\ln \varepsilon$ in view of (56). The specific heat diverges therefore logarithmically in four space dimensions.

In the canonical ensemble the temperature is one of the natural variables. The Gaussian model is not defined for a temperature $T$ below the value $T_{\mathrm{c}}=d J$. The microcanonical ensemble on the other hand has the energy as one of its natural variables. As demonstrated above the Gaussian model in dimensions $d \leq 2$ is defined for all possible energies although the conjugate variable temperature cannot be smaller than the value $T_{\mathrm{c}}$. For dimensions $d>2$ there exists a finite critical energy $e_{\mathrm{c}}$ and the Gaussian model in the microcanonical formalism is not defined for energies $e$ below the critical value. In the case of an algebraically diverging specific heat the microcanonical and the canonical critical exponents are related via equation (34). This relation is indeed satisfied by the critical exponents of the Gaussian model in $2<d<4$ dimensions. In four dimensions the specific heat diverges logarithmically in both approaches. For dimensions larger than four the specific heat stays finite and the corresponding critical exponent is zero. The singular behaviour of the specific heat of the Gaussian model in the various dimensions nicely demonstrate the discussed general interrelation of the scaling properties of microcanoncial and canonical physical quantities at phase transitions points.

\subsection{Spherical model}

The spherical model exhibits a continuous phase transition for dimensions larger than two with critical exponents that can be determined analytically both in the canonical ensemble [37, 39] and in the microcanonical ensemble [43, 44]. The canonical critical exponent of the specific heat is given by $\alpha=(d-4) /(d-2)$ for dimensions $2<d \leq 4$ and is in particular negative, suggesting that the divergence of the microcanonical specific heat is characterised by the same exponent. This is indeed found by analysing the critical properties in the entropy formalism [43]. For dimensions $d>4$ the specific heat shows a jump singularity. The order parameter is characterised by the exponent $\beta=1 / 2$ for all dimensions $d>2$ near the critical point again for both the canonical and the microcanonical ensemble.

\section{Corrections to scaling in the entropy formalism}

In this subsection the correction to scaling in the microcanonical ensemble is briefly considered for the reduced entropy formalism from a heuristic point of view. The starting point is again the Gibbs free energy which is of the form

$$
g(t) \stackrel{t \rightarrow 0}{\sim}-s_{\mathrm{c}} t-\frac{P_{ \pm}}{2-\alpha}|t|^{2-\alpha}\left(1+\frac{2-\alpha}{2-\alpha+\theta} \frac{Q}{P_{ \pm}}|t|^{\theta}\right)
$$

near the critical point $t=0$. As the exponent $\theta$ is assumed to be positive the correction to scaling term characterised by $\theta$ vanishes in the limit of small reduced temperatures $t$. Note again that the exponent $\theta$ 
is approximately 0.5 for systems of the universality class of the three-dimensional Ising model.

In section 4 the reduced entropy $\sigma(t)$ was inverted for the limit $t \rightarrow 0$. In this asymptotic limit the correction term can be neglected leading to the expression (26) for $t(\sigma)$. The expression for $t(\sigma)$ can now be improved by including higher order terms so that the functional equation (27) is satisfied also for the next correction term (compare (28)). Focusing only on positive reduced temperatures $t$ - and therefore positive reduced energies $\varepsilon$ and positive reduced entropies $\sigma$ - one gets the expression

$$
t(\sigma) \stackrel{\sigma \geq 0}{=} P_{+}^{\frac{1}{\alpha-1}} \sigma^{\frac{1}{1-\alpha}}-\frac{Q}{1-\alpha}\left(\frac{1}{P_{+}}\right)^{\frac{2-\alpha+\theta}{1-\alpha}} \sigma^{\frac{1+\theta}{1-\alpha}}+\ldots
$$

The Legendre transformation (24) now yields the reduced internal energy

$$
\varepsilon(\sigma) \stackrel{\sigma \geq 0}{=} T_{\mathrm{c}} \sigma+R_{+} \sigma^{\frac{2-\alpha}{1-\alpha}}+U_{+} \sigma^{\frac{2-\alpha+\theta}{1-\alpha}}+\ldots
$$

where the coefficient $U_{+}$is given by

$$
U_{+}=-\frac{Q}{2-\alpha+\theta}\left(\frac{1}{P_{+}}\right)^{\frac{2-\alpha+\theta}{1-\alpha}}
$$

The entropy is now obtained by inverting expression (59) for small $\sigma$. The asymptotic series (32) for the function $\sigma(\varepsilon)$ satisfies the functional equation $\varepsilon\left(\sigma\left(\varepsilon^{\prime}\right)\right)=\varepsilon^{\prime}$ only in the asymptotic limit of vanishing $\varepsilon^{\prime}$ :

$$
\varepsilon\left(\sigma\left(\varepsilon^{\prime}\right)\right)=\varepsilon^{\prime}+U_{+}\left(\frac{1}{T_{\mathrm{c}}}\right)^{\frac{2-\alpha+\theta}{1-\alpha}} \varepsilon^{\prime \frac{2-\alpha+\theta}{1-\alpha}}-\frac{2-\alpha}{1-\alpha} R_{+}^{2}\left(\frac{1}{T_{\mathrm{c}}^{2}}\right)^{\frac{2-\alpha}{1-\alpha}} \varepsilon^{\frac{3-\alpha}{1-\alpha}}+\ldots
$$

The correction term to the functional equation for the inverse $\sigma(\varepsilon)$ is of order $(2-\alpha+\theta) /(1-\alpha)$ if the exponent positive $\theta$ is small enough so that the term of order $(3-\alpha) /(1-\alpha)$ can be neglected (which is the case for the three-dimensional Ising model, for example). In this case the extended asymptotic series

$$
s(\varepsilon) \stackrel{\varepsilon \geq 0}{=} s_{\mathrm{c}}+\frac{1}{T_{\mathrm{c}}} \varepsilon-\frac{R_{+}}{T_{\mathrm{c}}}\left(\frac{1}{T_{\mathrm{c}}}\right)^{\frac{2-\alpha}{1-\alpha}} \varepsilon^{\frac{2-\alpha}{1-\alpha}}-\frac{U_{+}}{T_{\mathrm{c}}}\left(\frac{1}{T_{\mathrm{c}}}\right)^{\frac{2-\alpha+\theta}{1-\alpha}} \varepsilon^{\frac{2-\alpha+\theta}{1-\alpha}}+\ldots
$$

and hence

$$
\sigma(\varepsilon) \stackrel{\varepsilon \rightarrow 0^{+}}{\sim} \frac{1}{T_{\mathrm{c}}} \varepsilon-\frac{R_{+}}{T_{\mathrm{c}}}\left(\frac{1}{T_{\mathrm{c}}}\right)^{\frac{2-\alpha}{1-\alpha}} \varepsilon^{\frac{2-\alpha}{1-\alpha}}\left(1+\frac{U_{+}}{R_{+}}\left(\frac{1}{T_{\mathrm{c}}}\right)^{\frac{\theta}{1-\alpha}} \varepsilon^{\frac{\theta}{1-\alpha}}\right)
$$

for $\sigma(\varepsilon)$ satisfies the functional equation for the inversion of $\varepsilon(\sigma)$ up to the correction term of order $(2-\alpha+\theta) /(1-\alpha)$. The exponent $\theta$ of the correction to scaling term in the canonical ensemble is therefore also renormalised by the denominator $1-\alpha$ when going to the microcanonical ensemble. As this denominator is smaller than one $(\alpha \in] 0,1[)$ the next to leading corrections for small deviations from the critical point are qualitatively suppressed in the microcanonical ensemble compared to the canonical ensemble.

\section{Summary}

The properties of phase transitions in the entropy formalism can be formulated in terms of scaling relations for the entropy of the infinite system. For systems with an algebraically diverging specific 
heat at the continuous phase transition point these scaling relations have been demonstrated to be direct consequences of the corresponding well-established scaling properties of the Gibbs free energy. This connection extends the Hankey-Stanley theorem to thermodynamic potentials which are related to each other by partial inversions. Moreover, the corrections to scaling in the entropy formalism can also be linked to the corresponding corrections in the description based on the Gibbs free energy. It turns out that the corrections to scaling are suppressed in the entropy formalism. The considerations show that the critical exponents describing the singular behaviour in the microcanonical description are renormalised with respect to the canonical exponents. The discussion of a logarithmic specific heat and of a specific heat with a cusp singularity showed that the microcanonical and the canonical critical exponents are identical in these two cases.

\section{Acknowledgements}

The author is much obliged to Alfred Hüller and Michel Pleimling for many stimulating discussions and helpful comments on the manuscript. Fruitful discussions with Michael Schütz are also kindly acknowledged. The author also thanks the Deutsche Forschungsgemeinschaft for financial support (SFB 613).

\section{References}

1. Stanley, H. E. Introduction to Phase Transitions and Critical Phenomena; Oxford University Press: New York and Oxford, 1972; pp 175-190.

2. Goldenfeld, N. Lectures on Phase Transitions and the Renormalization Group; Addison-Wesley: Reading, 1992; pp 201-228.

3. Hankey, A.; Stanley, H. E. Systematic Application of Generalized Homogeneous Functions to Static Scaling, Dynamic Scaling, and Universality. Phys. Rev. B 1972, 6, 3515-3542.

4. Callen, H. B. Thermodynamics and an Introduction to Thermostatistics; Wiley \& Sons: New York, 1960; pp 85-102.

5. Desai, R. C.; Heermann, D. W.; Binder, K. Finite-Size Scaling in a Microcanonical Ensemble. J. Stat. Phys. 1988, 53, 795-823.

6. Brown, F. R.; Yegulalp, A. Microcanonical Simulation of First-Order Phase Transitions in Finite Volumes. Phys. Lett. A 1991, 155, 252-256.

7. Hüller, A. First Order Phase Transitions in the Canonical and the Microcanonical Ensemble. Z. Phys. B 1994, 93, 401-405.

8. Schmidt, M. MC-Simulation of the 3D, $q=3$ Potts Model. Z. Phys. B 1994, 95, 327-330.

9. Promberger, M.; Hüller, A. Microcanonical Analysis of a Finite Three-Dimensional Ising System. Z. Phys. B 1995, 97, 341-344.

10. Ota, S. B.; Ota, S. Microcanonical Monte Carlo Simulation of the First-Order Transition in the Two-Dimensional Potts Model. J. Phys.: Condes. Matter 2000, 12, 2233-2243.

11. Kastner, M.; M. Promberger, M.; Hüller, A. Microcanonical Finite-Size Scaling. J. Stat. Phys. 2000, 99, 1251-1264.

12. Gross, D. H.E. Microcanonical Thermodynamics: Phase Transitions in "small" Systems; World 
Scientific: Singapore, 2001.

13. Ispolatov, I.; Cohen, E. G. D. On First-Order Phase Transitions in Microcanonical and Canonical Non-Extensive Systems. Physica A 2001, 295, 475-487.

14. Hüller, A.; Pleimling, M. Microcanonical Determination of the Order Parameter Critical Exponent. Int. J. Mod. Phys. C 2002 13, 947-956.

15. Behringer, H. Symmetries of Microcanonical Entropy Surfaces. J. Phys. A: Math. Gen. 2003, 36, 8739-8751.

16. Behringer, H. Microcanonical Entropy for Small Magnetizations. J. Phys. A: Math. Gen. 2004, 37 , 1443-1458.

17. Pleimling, M.; Behringer, H.; Hüller, A. Microcanonical Scaling in Small Systems. Phys. Lett. A 2004, 328, 432-436.

18. Pleimling, M.; Behringer, H. Microcanonical analysis of small systems. Phase Transitions 2005, 78, 787-797.

19. Behringer, H.; Pleimling, M.; Hüller, A. Finite-size behaviour of the microcanonical specific heat. J. Phys. A: Math. Gen. 2005, 38, 973-985.

20. Behringer, H.; Pleimling, M. Continuous phase transitions with a convex dip in the microcanonical entropy. Phys. Rev. E 2006, 74, 011108.

21. Barré, J.; Mukamel, D.; Ruffo, R. Inequivalence of ensembles in a system with long-range Interactions. Phys. Rev. Lett. 2001, 87, 030601.

22. Leyvraz, F.; Ruffo, S. Ensemble inequivalence in systems with long-range interactions. J. Phys. A 2002, 35, 285-294.

23. Bouchet, F.; Barré, J. Classification of phase transitions and ensemble inequivalence, in systems with long range interactions. J. Stat. Phys. 2005, 118, 1073-1105.

24. Costeniuc, M.; Ellis, R. S.; Touchette, H.; Turkington, B. The generalized canonical ensemble and its universal equivalence with the microcanonical ensemble. J. Stat. Phys. 2005, 119, 1283-1329.

25. Costeniuc, M.; Ellis, R. S.; Touchette, H.; Turkington, B. Generalized canonical ensembles and ensemble equivalence. Phys. Rev. E 2006, 67, 026105.

26. Casetti, L.; Kastner, M. Partial equivalence of statistical ensembles and kinetic energy. Physica $A$ 2007, 384, 318-334.

27. Junghans, C.; Bachmann, M.; Janke, W. Microcanonical analyses of peptide aggregation processes. Phys. Rev. Lett. 2006, 97, 218103.

28. Chen, T.; Lin, X.; Liu, Y.; Liang, H. Microcanonical analysis of association of hydrophobic segments in a heteropolymer. Phys. Rev. E 2007, 76, 046110.

29. Junghans, C.; Bachmann, M.; Janke, W. Thermodynamics of peptide aggregation processes: An analysis from perspectives of three statistical ensembles. J. Chem. Phys. 2008, 128, 085103.

30. Dorlas, T. C. Statistical Mechanics - Fundamentals and Model Solutions; Insitute of Physics Publishing: Bristol and Philadelphia, 1999; p 120.

31. Wegner, F. J. Corrections to Scaling Laws. Phys. Rev. B 1972, 5, 4529-4536.

32. Guida, R.; Zinn-Justin, J. Critical Exponents of the N-Vector Model. J. Phys. A: Math. Gen. 1998, 31, 8103-8121.

33. Hasenbusch, M. A Monte Carlo Study of Leading Order Scaling Corrections of $\phi^{4}$ Theory on a 
Three-Dimensional Lattice. J. Phys. A: Math. Gen. 1999, 32, 4851-4865.

34. Fisher, M. E. Renormalization of Critical Exponents by Hidden Variables. Phys. Rev. 1968, 176, 257-272.

35. Behringer, H.; Pleimling, M.; Hüller, A. Phase Transitions in a Cluster Molecular Field Approximation. Eur. Phys. J. B 2003, 31, 81-93.

36. Richter, A.; Pleimling, M.; Hüller, A. The density of states of classical spin systems with continuous degrees of freedom. Phys. Rev. E 2005, 71, 056705.

37. Berlin, T. H.; Kac, M. The spherical model of a ferromagnet. Phys. Rev. 1952, 86, 821-835.

38. Lewis, H. W.; Wannier, G. H. Spherical model of a ferromagnet. Phys. Rev. 1952, 88, 682-683.

39. Baxter, R. J. Exactly solved models in statistical mechanics; Academic Press: London, 1982; pp 60-71.

40. Thompson, C. J. Classical Equilibrium Statistical Mechanics; Clarendon Press: Oxford, 1988; pp 158-164.

41. Goldenfeld, N. Lectures on Phase Transitions and the Renormalization Group; Addison-Wesley: Reading, 1992; pp 353-361.

42. Morandi, G.; Napoli, F.; Ercolessi, E. Statistical Mechanics. An Intermediate Course; World Scientific: Singapore, 2001; pp 414-421.

43. Behringer, H. Critical properties of the spherical model in the microcanonical formalism. J. Stat. Mech.: Theory Exp. 2005, 06014.

44. Risau-Gusman, S.; Ribeiro-Teixeira, A. C.; Stariolo, D. A. Topology and phase-transitions: The case of the short range spherical model. J. Stat. Phys. 2006, 124, 1231-1253.

45. Joyce, G. S. Critical properties of the spherical model. In Phase Transitions and Critical Phenomena, Vol. 2; Domb, C.; Green M. S., eds.; Academic Press: London, 1972; p 397.

(c) 2008 by the authors; licensee Molecular Diversity Preservation International, Basel, Switzerland. This article is an open-access article distributed under the terms and conditions of the Creative Commons Attribution license (http://creativecommons.org/licenses/by/3.0/). 